\title{
Space charge induced resonance excitation in high intensity rings
}

\author{
S. Cousineau and S. Y. Lee \\ Indiana University, Bloomington, Indiana 47405 \\ J. A. Holmes and V. Danilov \\ Oak Ridge National Laboratory, Oak Ridge, Tennessee 37831 \\ A. Fedotov \\ Brookhaven National Laboratory, Upton, New York 11973 \\ (Received 4 September 2002; published 26 March 2003)
}

\begin{abstract}
We present a particle core model study of the space charge effect on high intensity synchrotron beams, with specific emphasis on the Proton Storage Ring (PSR) at Los Alamos National Laboratory. Our particle core model formulation includes realistic lattice focusing and dispersion. We transport both matched and mismatched beams through real lattice structure and compare the results with those of an equivalent uniform-focusing approximation. The effects of lattice structure and finite momentum spread on the resonance behavior are specifically targeted. Stroboscopic maps of the mismatched envelope are constructed and show high-order resonances and stochastic effects that dominate at high mismatch or high intensity. We observe the evolution of the envelope phase-space structure during a high intensity PSR beam accumulation. Finally, we examine the envelope-particle parametric resonance condition and discuss the possibility for halo growth in synchrotron beams due to this mechanism.
\end{abstract}

DOI: $10.1103 /$ PhysRevSTAB.6.034205

PACS numbers: 29.27.Bd

\section{INTRODUCTION}

The importance of space charge as a cause of emittance growth, halo formation, and beam loss has long been accepted. With many future high intensity machines operating in the energy regime of several hundred $\mathrm{MeV}$ to a few $\mathrm{GeV}$, the relevance of space charge physics has increased dramatically. In 1959, Kapchinskij and Vladimirskij (KV) introduced a self-consistent analytic model for space charge study [1], thus providing a framework for understanding the basic physics and consequences of space charge interactions in an idealized beam. Sacherer followed with analyses of the integer envelope resonance condition for coherent beam modes in the absence of structure resonances due to lattice periodicity [2]. The integer envelope resonance, often referred to as the coherent half-integer resonance, is associated with betatron tunes near integer or half-integer values. The treatment of the coherent mode resonances was later extended to periodic focusing systems and included envelope instabilities for second order beam modes [3].

The aforementioned studies are based on the broad class of particle core models (PCMs), which solve the equations of motion of the beam envelope. PCMs have been utilized both analytically and in computer tracking simulations to explain a number of resonance phenomena observed in high intensity machines [4-6]. Historically, these studies were performed for linac beams, where the peak density of particles is much greater than in rings. The effect of space charge on the synchrotron beam is a more recent concern, and analogous PCM studies are being undertaken for the ring beam environment [7]. In these studies, the particle core model has either been applied to idealized lattice structures and beam parameters, or the effects of the lattice have not been critically examined. To fully understand the effect of space charge on the high intensity synchrotron beam, both realistic beam parameters and lattice structure must be considered.

This paper presents a numerical PCM study of the space charge effect on high intensity synchrotron beams, motivated by experimentally observed space charge induced beam broadening and emittance growth in the Proton Storage Ring (PSR) at Los Alamos National Laboratory [8]. The PSR is approximately $90 \mathrm{~m}$ in circumference and is used for compression of an $800 \mathrm{MeV}$ proton beam. At the highest operating intensities, the PSR beam can encounter very strong space charge effects, making it an ideal candidate for studies of space charge induced resonant excitations. In earlier work, a number of particle-in-cell (PIC) simulations of the experimentally observed broadening were presented [9-11]. In the present study, we focus primarily on a particle core model description of space charge induced resonant excitations in a high intensity synchrotron beam. We treat the problem specifically for an intense space charge beam in the PSR lattice. Since the beam parameters are varied arbitrarily, not all of the computations are representative of the true PSR beam; results relevant to the real PSR machine are specifically identified. We discuss the resonance response of the envelope closed-orbit (matched envelope condition), the implications of envelope mismatch, and the possibility of halo generation due to the envelope-particle parametric resonance. The effects of the real lattice 
environment and finite momentum spread on the resonance structure are specifically targeted. Additionally, we examine the harmonic content of the PSR lattice and identify driving terms for the envelope resonant excitation.

The paper is organized as follows: In Sec. II, we describe the envelope resonance condition for the matched beam in the PSR ring; we compare a computational model that includes realistic lattice focusing and dispersion with a standard, uniform-focusing analytic model developed by Sacherer. In Sec. III, we extend our study to a nonlinear analysis of a mismatched beam core in the PSR ring. In Sec. IV, we examine the particle phase space under the influence of a mismatched core and reflect on the parametric resonance condition as a possible mechanism for emittance growth. In Sec. V we summarize.

\section{THE ENVELOPE INTEGER RESONANCE}

A thorough analysis of the integer envelope resonance condition was first presented by Sacherer in his dissertation thesis [2]. Since the envelope tune of a beam is approximately twice the betatron tune, integer envelope resonances can occur when the betatron tune nears integer or half-integer values. Sacherer exercised the KV formalism to derive a set of equations that govern the onset and behavior of these resonances. In his work, he obtained the following coupled $\mathrm{KV}$ envelope equations:

$$
\begin{aligned}
& \frac{d^{2} y}{d \phi^{2}}+\left[\nu_{y}^{2}+2 \nu_{y} \cdot \Delta \nu_{e y} \cos (n \phi)\right] y-\frac{\nu_{y}^{2}}{y^{3}}- \\
& \frac{4 r_{0} \lambda}{\gamma^{3} \beta^{2}} \frac{\nu_{y}^{2} \beta_{y}^{3 / 2}}{\sqrt{\epsilon_{y}}} \frac{1}{\sqrt{\beta_{x} \epsilon_{x}} x+\sqrt{\beta_{y} \epsilon_{y}} y}=0,
\end{aligned}
$$

and similarly for the horizontal plane, where $\nu_{y}$ represents the vertical bare tune, $\phi$ is the longitudinal coordinate with $\phi=\int_{s_{o}}^{s}\left[(d s) /\left(\nu_{y} \beta_{y}\right)\right], \Delta \nu_{e y} \cos (n \phi)$ with integer $n$ represents an ad hoc quadrupole error term, $\gamma$ and $\beta$ are relativistic factors, $\lambda$ is the beam particle line density, $r_{0}$ is the classical proton radius, $\beta_{x}$ and $\beta_{y}$ are the beta functions, and $\epsilon_{x}$ and $\epsilon_{y}$ are the rms emittances. The $y$ coordinate is scaled to a dimensionless variable, $y \rightarrow$ $\left(y / \sqrt{\beta_{y} \epsilon_{y}}\right)$, such that $y$ is normalized to the zero-space charge envelope. The model assumes a coasting beam for the longitudinal distribution, which is a good approximation when the bunch length is long compared to the width of the beam, as is the case for most synchrotrons. From left to right in Eq. (1), the first term is the acceleration term, the second term represents the contribution of magnetic focusing, the third term results from emittance factors, and the last term is due to the space charge force of the beam. In solving Eq. (1), Sacherer made two simplifying assumptions: (i) He ignored the oscillatory nature of the beta function by assuming a uniform-focusing lattice, and (ii) he assumed that the magnetic error term and space charge tune shifts were small enough to merit a perturbative approach. Temporarily ignoring the error term, his approximations lead him to the following coupled system of integral equations for the envelope:

$$
\begin{gathered}
x^{2}=\sqrt{1+A^{2}}+A \sin (u), \\
y^{2}=\sqrt{1+B^{2}} \pm B \sin (u), \\
A=\frac{w_{p}^{2}}{2 \nu_{x} \Delta \nu_{x}} \frac{b}{2 \pi} \int_{0}^{2 \pi} \frac{A+\sqrt{1+A^{2}} \sin (u)}{x(a x+b y)} d u, \\
B=\frac{w_{p}^{2}}{2 \nu_{y} \Delta \nu_{y}} \frac{a}{2 \pi} \int_{0}^{2 \pi} \frac{B \pm \sqrt{1+B^{2}} \sin (u)}{y(a x+b y)} d u,
\end{gathered}
$$

where $\quad a=\sqrt{\beta_{x} \epsilon_{x}}, \quad b=\sqrt{\beta_{y} \epsilon_{y}}, \quad w_{p}^{2}=\left[\left(2 N r_{o} R\right) /\right.$ $\left.\left(\pi a b \gamma^{3} \beta^{2}\right)\right], N$ is the number of particles in the beam, $R$ is the length of the ring, and $\Delta \nu_{x}$ and $\Delta \nu_{y}$ are the differences between the bare tune and the nearest underlying integer or half integer. Two solutions are obtained for the system: a symmetric mode and an antisymmetric mode. Both solutions represent periodic envelope trajectories or fixed points in the envelope phase space. Analytic results exist for some simplified cases [12], but for realistic parameters, such as for the PSR beam, the equations must be solved numerically. The main thrust of Sacherer's analysis is that, beyond a certain threshold incoherent tune shift, the beam envelope can undergo periodic oscillations about the zero-space charge envelope solution, and the amplitude of these oscillations increases nonlinearly with the level of space charge in the beam. An example of a vertical periodic resonant envelope oscillation over one turn of a PSR phase-advance-equivalent uniform-focusing lattice is shown in Fig. 1. One of the most notable results of his work is that the onset of the envelope resonance condition occurs at intensities beyond the incoherent stop band limit. In contrast with predictions derived from single particle dynamics, this implies that particles in the beam react collectively to the space charge force rather than individually [12].

Although the qualitative behavior of Sacherer's model has been verified both analytically and computationally $[3,4,13,14]$, the model is too simplified to describe a realistic beam scenario. The uniform-focusing structure represents a significant approximation, and a lattice structure that includes realistic focusing and dispersion will affect the beam dynamics, particularly the strength and onset of resonances. To this end, another more robust version of the particle core model, first presented by Lee and Okamota [15] and later extended to a computational framework by Holmes et al. [16], allows us to consider the effect of a real lattice, including dispersion. This alternative model was derived from an rms standpoint and does not include higher order moment contributions [16]. It has been implemented into the ACCSIM 


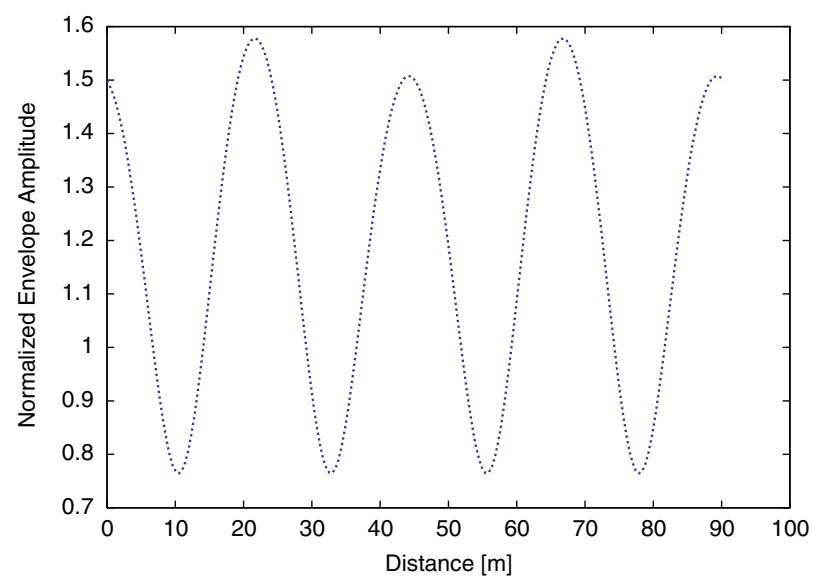

FIG. 1. (Color) Vertical beam envelope versus distance over one turn of a PSR phase-advance-equivalent uniform-focusing lattice. The envelope is normalized to the zero-space charge envelope, $\sqrt{\beta_{y} \epsilon_{y}}$.

particle tracking code [17], where the envelope motion including dispersion is solved by iterating the following equations:

$$
\begin{gathered}
a^{\prime \prime}-\frac{\epsilon_{x}^{2}}{a^{3}}+\left[K_{x}(s)-\frac{4 q^{2} \lambda}{A(A+B) \gamma^{2} \beta^{2} E_{0}}\right] a=0, \\
b^{\prime \prime}-\frac{\epsilon_{y}^{2}}{b^{3}}+\left[K_{y}(s)-\frac{4 q^{2} \lambda}{B(A+B) \gamma^{2} \beta^{2} E_{0}}\right] b=0, \\
D_{x}^{\prime \prime}(s)+\left[K_{x}(s)-\frac{4 q^{2} \lambda}{A(A+B) \gamma^{2} \beta^{2} E_{0}}\right] D_{x}(s)-\frac{1}{\rho}=0 .
\end{gathered}
$$

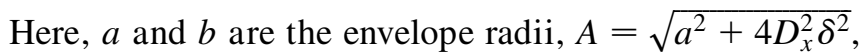
$B=b, D_{x}$ is the space charge adjusted dispersion function, $K_{x}(s)$ and $K_{y}(s)$ are the horizontal and vertical focusing functions, respectively, $\lambda$ is the beam particle line density, $E_{0}=\gamma m c^{2}$ is the reference energy of the beam, $\rho$ is the bending radius, and $\delta=\Delta p / p$ is the rms momentum spread, and the derivatives are taken with respect to the longitudinal coordinate, $s$. Again, the model is derived assuming a coasting beam for the longitudinal distribution. The closed-orbit solution is obtained by solving with periodic boundary conditions, and the equations restrict the applications to observable phenomena that are independent of higher than second order beam moments. The matched envelope including dispersion can be found by initializing $a, b$, and $D$ to their space charge-free values and iterating through the equations using a shooting method until convergence in phase space is achieved. As a comparison of this implementation with Sacherer's model, a beam with $\Delta p / p=0$ and parameters typical of the PSR beam was transported through a uniform-focusing channel with phase advance equivalent to the PSR lattice. Figure 2 shows the result, where the maximum of the envelope oscillation, normal-

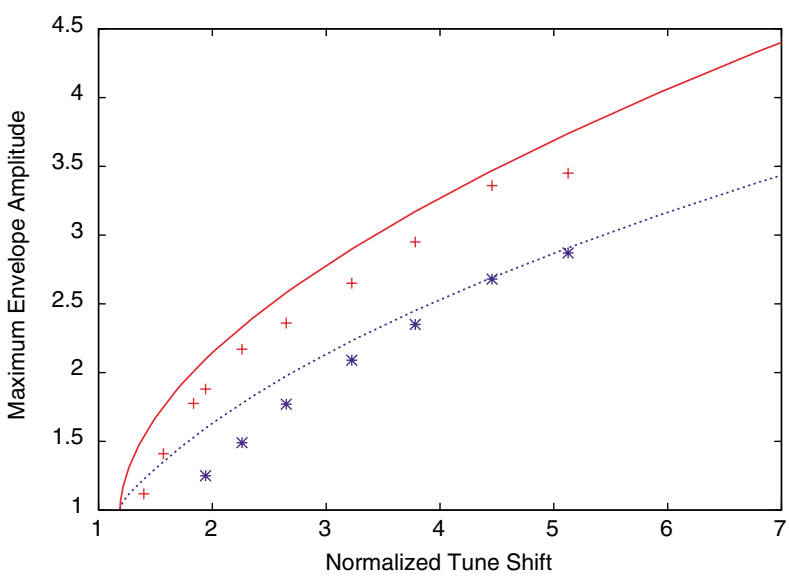

FIG. 2. (Color) The maximum normalized envelope amplitude versus the vertical space charge tune shift normalized to the fractional vertical bare tune (0.19). The solid (red) and dashed (blue) lines represent the predictions of Sacherer's model for the horizontal and vertical planes, respectively, and the red +'s and blue *'s represent the horizontal (with $\Delta p / p=0$ ) and vertical predictions, respectively, of the particle core model implemented in the ACCSIM tracking code.

ized to the zero space charge envelope, $\sqrt{\beta \epsilon_{\text {rms }}}$, is plotted against the space charge tune shift, normalized to the fractional vertical betatron tune $(0.19$, in this case). Overall, the agreement between the two models is good. It is not surprising to observe small differences in the results since Sacherer employed a perturbative approximation in his analysis, ignoring terms above first order, whereas the above model solves the exact equations numerically. Note that both models predict the onset of the resonance at intensities at least $20 \%$ greater than the incoherent stop band limit.

The core model in the ACCSIM code can be used to calculate the envelope motion of a beam in a realistic accelerator lattice. The PSR parameters shown in Table I are used, where the listed beam intensity is typical of a high intensity PSR beam accumulation scenario; the parameters in the table do not represent the baseline PSR operating parameters. Since the particle core model has uniform longitudinal density, whereas the real PSR beam does not, for our calculations we choose the intensity as

TABLE I. PSR parameters for a high intensity beam accumulation scenario. Emittances typical of the beam halfway through the accumulation.

\begin{tabular}{lc}
\hline \hline PSR lattice length & $90.26 \mathrm{~m}$ \\
Full PSR beam intensity & $4.37 \times 10^{13} \mathrm{protons}$ \\
Kinetic energy & $800 \mathrm{MeV}$ \\
Horizontal tune & 3.19 \\
Vertical tune & 2.19 \\
rms horizontal emittance & $2.58 \pi \mathrm{mm} \mathrm{mrad}$ \\
rms vertical emittance & $5.19 \pi \mathrm{mm} \mathrm{mrad}$ \\
\hline \hline
\end{tabular}


the peak intensity of the real PSR beam at any given stage of accumulation, i.e., we divide the average intensity by the PSR bunching factor, approximately 0.30 [11]. To study the effect of the space charge force, we arbitrarily vary the beam intensity from the value listed in the table. The emittances, however, are kept constant throughout the remainder of the discussion at the values given in Table I, unless otherwise specified. The emittances are representative of the PSR beam at approximately halfway through a high intensity beam accumulation. Therefore, since the intensity parameter will be varied while the emittance is kept fixed, not all of the calculations presented below are representative of the true PSR beam.

Figure 3 shows the maximum normalized envelope amplitude versus the normalized tune shift for the real PSR lattice and for a PSR phase-advance-equivalent uniform-focusing approximation. In order to isolate the effect of the lattice structure, we have removed dispersion by assuming zero energy spread for the beam in this instance; the effect of finite energy spread is discussed later in this section. Whereas the resonance threshold for the uniform lattice is clearly defined, the real lattice broadens the response in tune space significantly. In fact, a small amount of oscillation is observed even before the normalized tune shift value of $\nu_{\text {norm }}=1$, that is, before the incoherent stop band limit. Similar broadening was observed by Sacherer to result from the ad hoc error driving term in the envelope Eq. (1). The real PSR lattice contains error terms resulting from lattice harmonics. An analytic example which demonstrates the role of the lattice in the periodic envelope resonance is given in Appendix A.

Another effect noticeable in Fig. 3 is that no closedorbit solutions exist for the real lattice beyond the normalized horizontal tune shift of $\nu_{\text {norm }} \geq 2.5$. In this region, a true closed-orbit solution cannot be found. It is possible to find a solution that appears periodic over one

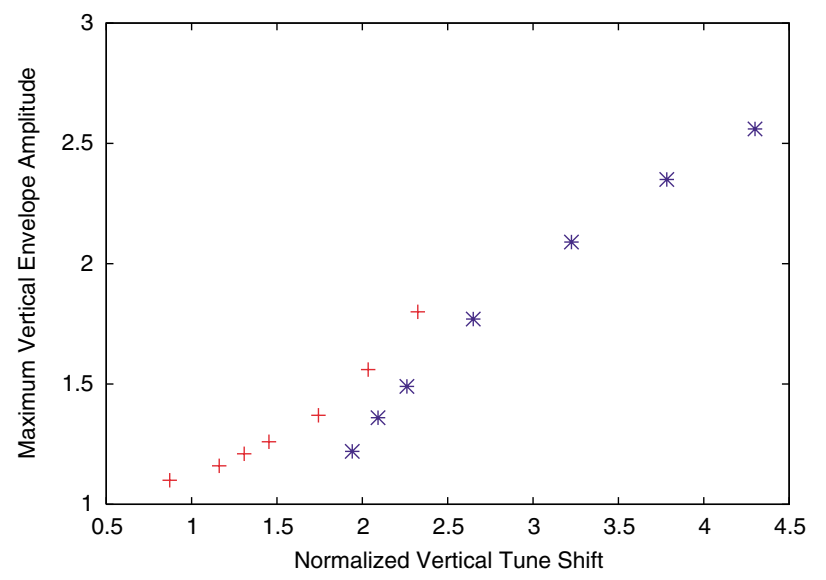

FIG. 3. (Color) Vertical coherent resonance response curves for the PSR lattice (red +'s), and for an equivalent uniformfocusing approximation (blue $*$ 's). turn of the ring, but this solution does not remain stable over multiple turns, as would a true closed orbit. This closed-orbit instability coincides with the presence of lattice driving harmonics; the smooth approximation which does not contain the harmonics produces stable closed-orbit solutions for all values of normalized tune shift.

For the PSR lattice with bare vertical tune $\nu_{y}=2.19$, the matched resonant envelope is excited by an $n=4$ lattice harmonic. The harmonic content of the lattice is embodied in the beta functions. Figure 4 shows the harmonic spectrum of the PSR vertical beta function for the working point under study. The tenfold PSR lattice inherently gives rise to strong harmonics at multiples of 10 . However, the lattice is not perfectly symmetric, and the break in symmetry allows for the existence of other harmonics. In fact, the strongest harmonic that is not a multiple of 10 is the $n=4$ harmonic. Therefore, the
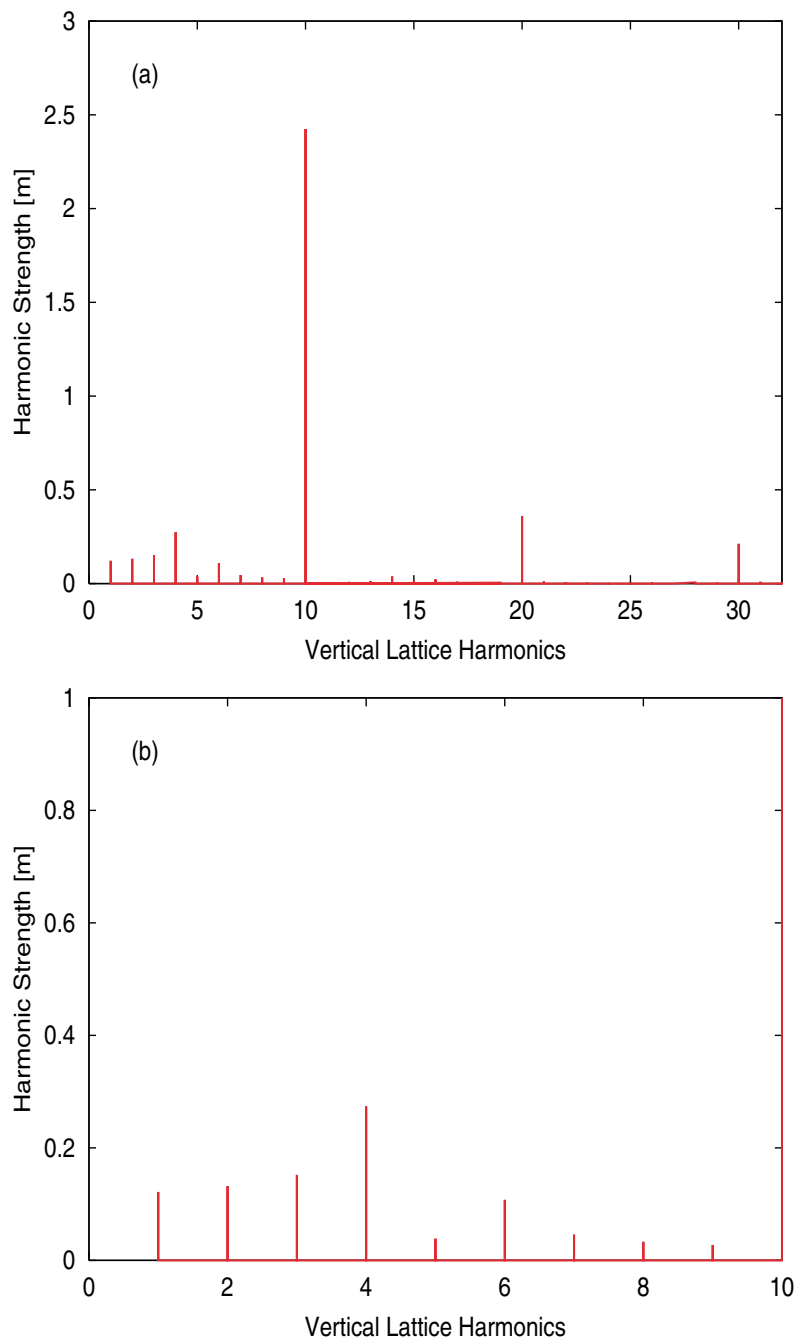

FIG. 4. (Color) The harmonic spectrum of the PSR vertical beta function. Plot (a) shows a large portion of the spectrum, while plot (b) zooms in on the low-order harmonics. 
driving term for an envelope quadrupole-mode resonance is built into the bare lattice structure, and it should be no surprise that we observe the manifestation of this phenomenon experimentally [11].

It is also important to assess the effect of dispersion on the behavior of the matched envelope resonance. The PSR lattice has nonzero horizontal dispersion throughout the entire lattice and zero dispersion in the vertical direction. Simulations were performed using the beam parameters in Table I, and assuming an rms momentum spread of $\Delta p / p \cong 2 \times 10^{-3}$, derived from particle tracking simulations in Ref. [11]. Results for both the dispersive horizontal plane and the nondispersive vertical plane are shown in Fig. 5. In the horizontal plane, the dispersion significantly decreases the resonance response because it increases the average beam size, thus weakening the
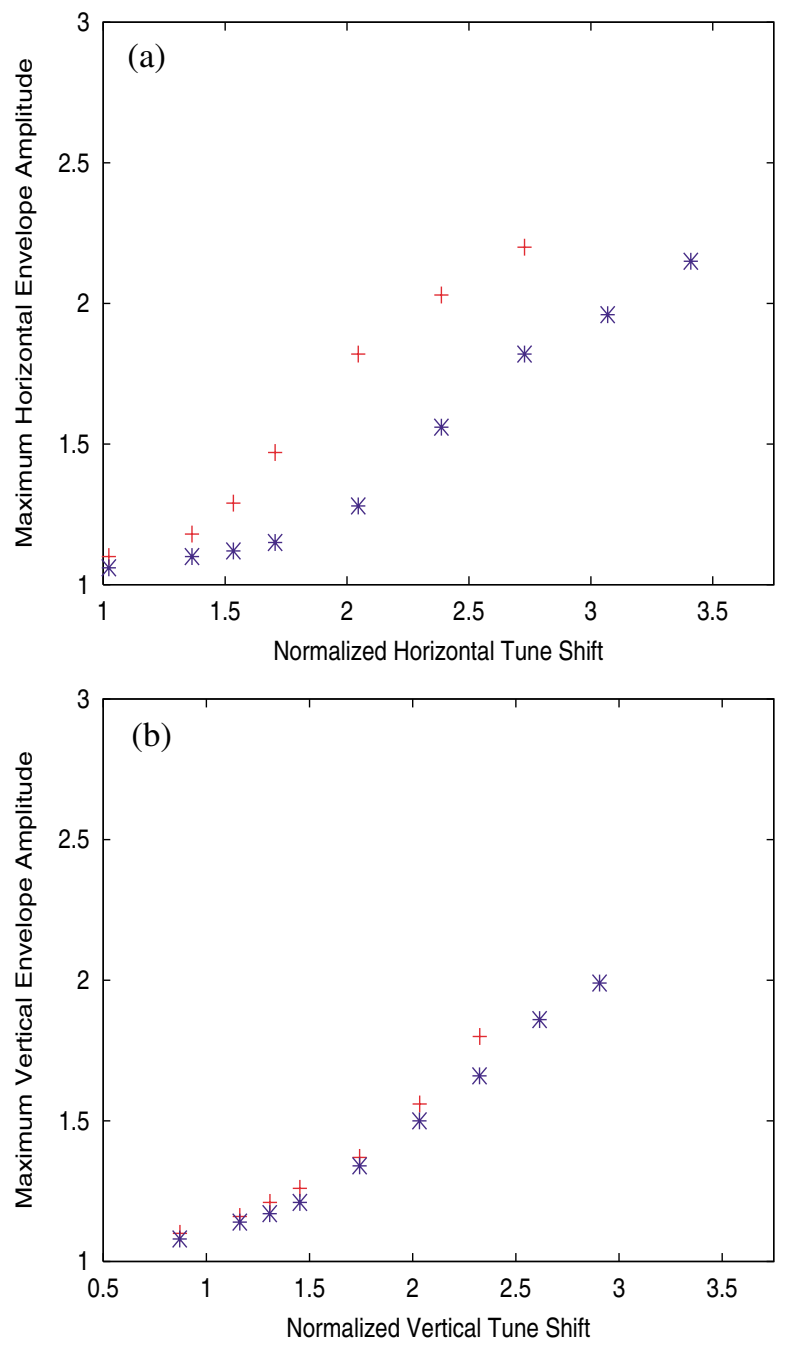

FIG. 5. (Color) Coherent resonance response curves in the horizontal direction (a) and the vertical direction (b) with and without energy spread. The (red) +'s represent the response without dispersion, and the (blue) *'s represent the response with dispersion.

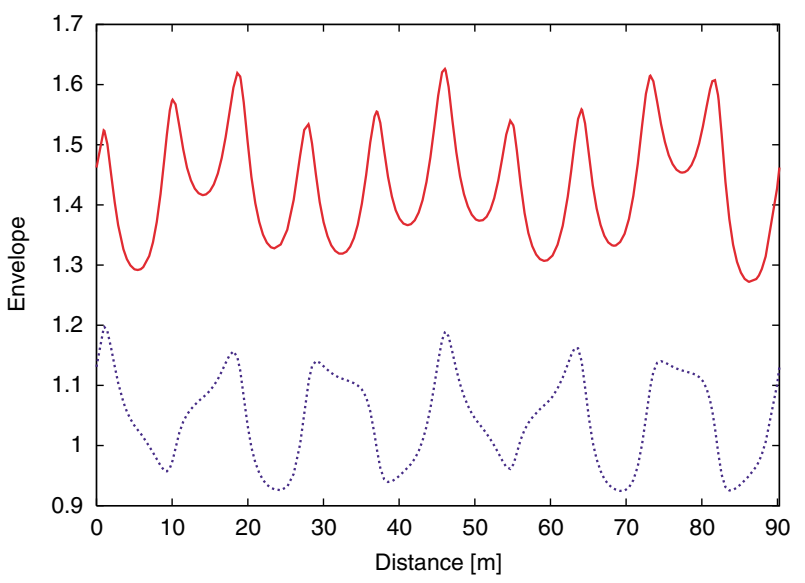

FIG. 6. (Color) Beam envelope versus distance along the PSR lattice, plotted with and without the dispersion term. The (red) solid line is the envelope plotted with the dispersion, and the (blue) dashed line is the envelope plotted without the dispersion.

strength of the space charge force. The small reduction of the response in the vertical direction is likewise due to a weakening of the space charge when the dispersion is included.

Another effect arising from the dispersion term is that it complicates the behavior of the envelope function, making the resonance signature difficult to detect. Depending on the momentum deviation, the envelope motion may be dominated by the dispersion function, and the resonance is a small oscillation about this function. As an example, a calculation is performed using the parameters in Table I and incorporating a finite rms momentum spread, $\Delta p / p \cong 2 \times 10^{-3}$. Though dispersion was used in the calculation, for diagnostic purposes the dispersion contribution can be subtracted out of the result and the envelope motion over one turn of the PSR ring can be plotted with and without dispersion, as shown in Fig. 6. Notice that the dispersion term with superperiod ten completely masks the six-period horizontal envelope resonance. Although our particle core model can decouple the resonance signal from the dispersion, more realistic simulations, such as PIC simulations, do not provide this luxury. For this reason, unless one seeks to study the dispersion term specifically, it is much simpler to analyze the coherent motion of a beam in the plane with zero dispersion.

\section{THE EFFECT OF MISMATCH ON THE ENVELOPE}

The discussion of the envelope resonance condition thus far treats only closed-orbit solutions of the envelope equation. In this case, the beam is perfectly matched and the envelope varies periodically with respect to the lattice, undergoing $n$ modulations around the circumference, and returning to the same phase-space point on consecutive turns. The envelope of a beam which is not 
perfectly matched to the lattice will execute phase-space oscillations about this closed orbit. A higher order expansion of the last term in Eq. (1), combined with lattice terms, can excite higher order envelope-lattice resonances. With the core model implemented in ACCSIM, it is possible to observe this behavior by applying an initial perturbation and tracking the subsequent evolution of the mismatched, free envelope. For our computations, we define a mismatch parameter, $M$, as

$$
M \equiv \frac{R-R_{c}}{R_{c}}
$$

where $R_{c}$ is the initial value of the closed-orbit solution of the envelope for either plane, and $R$ is the initial value of the envelope coordinate $(X$ or $Y)$. The condition for the matched beam is $M=0$. The mismatched beam envelope will encounter a resonance with the lattice whenever its tune nears a rational number. An analytic example which highlights the resonance physics in this situation was presented by Lee and Riabko [18]. The occurrence of such resonances depends sensitively on the strength of the space charge, the magnitude of the mismatch parameter, the presence of appropriate lattice driving terms, and the unperturbed betatron tunes of the ring lattice. A scan over mismatch parameters at any given intensity can be used to produce a one-turn (stroboscopic) map of the envelope phase space.

Figure 7 shows a stroboscopic map of the mismatched vertical PSR envelope in the real PSR lattice structure, as well as the envelope tune over the range of mismatch parameters. The beam intensity here is approximately one-quarter of the full PSR intensity, i.e., a fourth of the way through the accumulation cycle of the $4.37 \times$ $10^{13}$ proton beam, and other beam parameters are as in Table I. The resonance structure is explored by plotting the envelope phase-space coordinates at the same point in the ring on consecutive turns. The fixed point in the center of the map represents the closed-orbit solution of the envelope discussed in the previous section, and the alternating colors represent different values of initial envelope mismatch. The fixed point has $M=0$, and the direction of increasing mismatch is radially outward from this point. The largest resonance seen in the map is a $4: 1$ resonance, corresponding to an envelope tune of $\nu_{e}=$ 4.25 and mismatch parameters in the range $M \cong$ $0.2, \ldots, 0.3$. Such a strong, low order resonance is dangerous if encountered because it allows the beam quick access to a broad range of mismatch. Moving beyond the 4:1 resonance to higher mismatch, the envelope undergoes larger amplitude oscillations and spends less time in the dense, high space charge state. The envelope tune tends toward the zero-space charge limit of twice the bare tune, i.e., $\nu_{e}=2 \nu_{y}=4$.38. Progressing from $\nu_{e}=$ 4.25 towards this upper limit, several weaker, higher order resonances are observed. Specifically, in order of
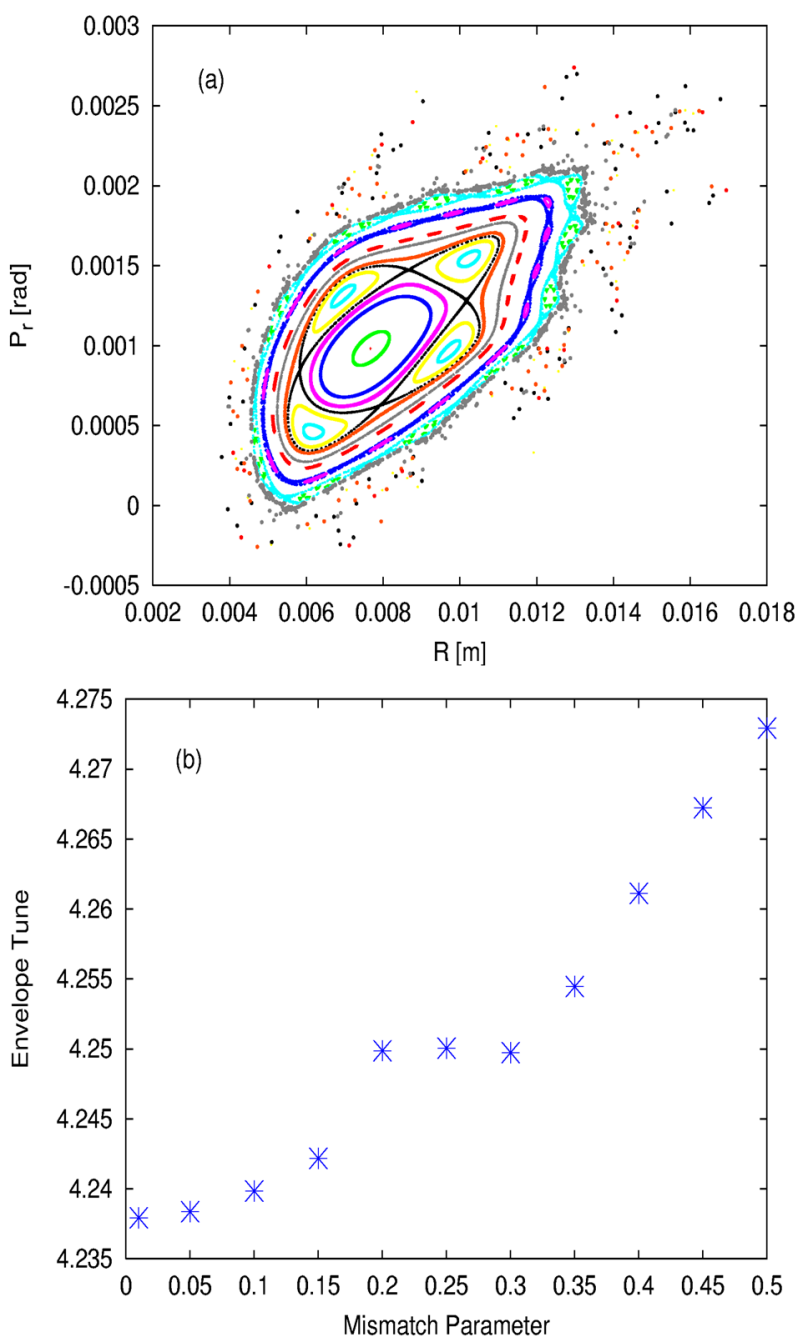

FIG. 7. (Color) (a) Stroboscopic map of the vertical envelope in the PSR lattice for a beam with intensity equal to one-quarter of the full PSR beam intensity listed in Table I. The colors represent varying levels of mismatch. (b) Envelope tune versus the mismatch values used in (a).

increasing mismatch, we see the 23:6 resonance $\left(\nu_{e}=\right.$ 4.261), the 15:4 resonance $\left(\nu_{e}=4.267\right)$, and the 11:3 resonance $\left(\nu_{e}=4.273\right)$. Increasing the mismatch further, we observe that the higher order resonances are encompassed in a stochastic band arising from overlapping of resonances. Stochasticity exists for all levels of mismatch, but is exponentially small close to the fixed point. Once the mismatch is high enough for the beam to enter the outer stochastic band, the evolution is chaotic and the envelope behavior is no longer predictable. It is likely that a beam would undergo a major relaxation in this region, but the physics of such a process is beyond the scope of our model.

As we increase the intensity of the beam toward the PSR full beam intensity, the envelope tune is further depressed, and resonance islands seen at lower intensities move outward into the stochastic band while new sets of 
islands emerge close to the fixed point. Figure 8 shows a stroboscopic map of the envelope at just over half of the highest PSR beam intensity, again for the parameters given in Table I. The emittance and intensity parameters used in this map represent a good approximation to the real PSR beam halfway through a high intensity accumulation cycle. Note that the outer stochastic band has broadened considerably, and that stochastic bands near the fixed point are now visible. At low mismatch the 8:1 resonance $\left(\nu_{e}=4.125\right)$ lies very close to a small chaotic band, at moderate mismatch the 7:1 $\left(\nu_{e}=4.143\right)$ resonance islands are visible, and at high mismatch the separatrix of the 6:1 resonance $\left(\nu_{e}=4.167\right)$ is entirely stochastic [19]. This is the highest intensity for which a well-defined fixed point surrounded by closed surfaces can be found using the beam emittances given in Table I. At higher intensities, we can construct a map for the high
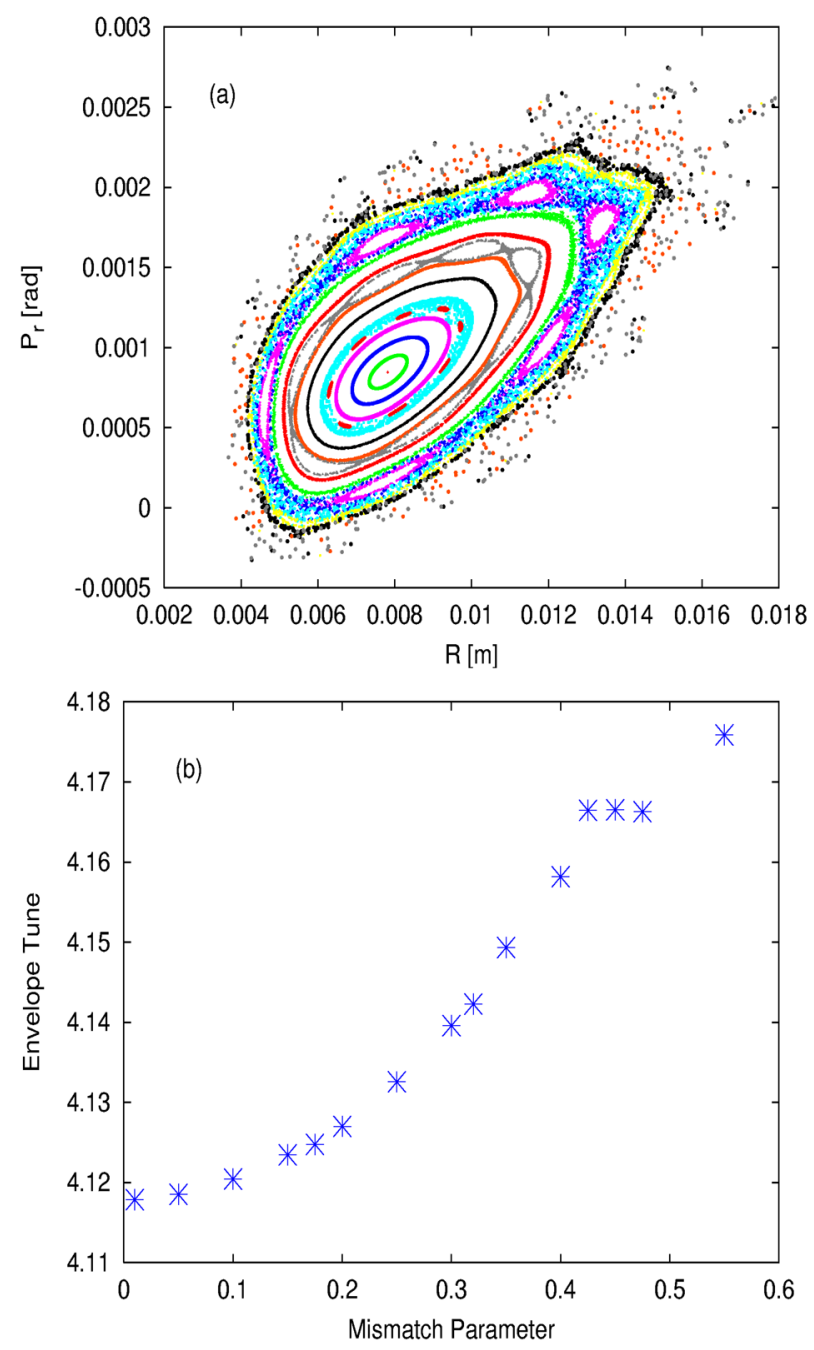

FIG. 8. (Color) (a) Stroboscopic map of the envelope in the PSR lattice for a beam with intensity equal to one-half of the full PSR beam intensity listed in Table I. The colors represent varying levels of mismatch. (b) Envelope tune versus the mismatch. mismatch region, where the envelope tune is above $\nu_{e} \cong$ 4.11. However, for small mismatch parameters resulting in envelope tunes below this value, the envelope is too close to closed-orbit instability discussed in the previous section. In these simulations, the width of the unstable region is very large and solutions cannot be obtained for $\Delta \nu_{\text {instability }} \leq 0.11$.

It is interesting to consider the evolution of the envelope phase space of the PSR beam during a high intensity beam accumulation. Experimentally, the PSR beam emittance grows in excess of injection painting effects during the high intensity beam accumulation [11]. Figure 8 is approximately representative of the PSR beam halfway through a high intensity beam accumulation cycle. Using the beam intensity listed in Table I, and emittances derived from PIC simulations in [11], a stroboscopic map for the PSR beam at the end of the high intensity accumulation is constructed and shown in Fig. 9. Although the beam intensity has more than doubled between Figs. 8 and 9, the maps and corresponding envelope tune plots are very similar, with the same basic resonance structure and tune range. At both stages in the accumulation, the nearly matched PSR beam resides near the closed-orbit instability; the emittance growth of the beam coincides with the envelope nearing the edge of the instability, and the growth rate balances the intensity increase to maintain an envelope tune slightly greater than the instability threshold. The connection between the instability and the emittance growth is not yet clear, except to say that the high intensity PSR beam undergoes emittance growth when the envelope tune nears a certain threshold value. Since the phase space of the envelope in the vicinity of $\nu_{e}=4.0$ is influenced most strongly by the $n=4$ lattice harmonic, a reduction of the harmonic in the PSR lattice could prove effective in treating the closed-orbit instability and the space charge induced emittance growth in the beam. Further study on this topic is underway.

In keeping with the analysis of the previous section, it is interesting to explore the effects of both the focusing structure and dispersion on the mismatched beam. We investigate the focusing structure first. Figure 10 reproduces the stroboscopic map and tune plot of Fig. 7, this time with a smooth lattice approximation in place of the real PSR lattice. In this approximation, the matched envelope is a constant, so the fixed point has envelope momentum $P_{r}=0$. At higher mismatch, the $4: 1$ resonance is seen, but its strength, as measured by the width of the islands, is zero; the lattice no longer contains the harmonics necessary to drive the resonance. Additionally, since the smooth lattice has an averaging influence on the envelope, the stochastic bands that dominate the high mismatch regime in Fig. 7 do not exist here. Because of the lack of lattice harmonics, a well-defined fixed point in the envelope phase space surrounded by closed surfaces can be found for all beam intensities, including the region near $\nu_{e}=4$, which is occupied by the closed-orbit 

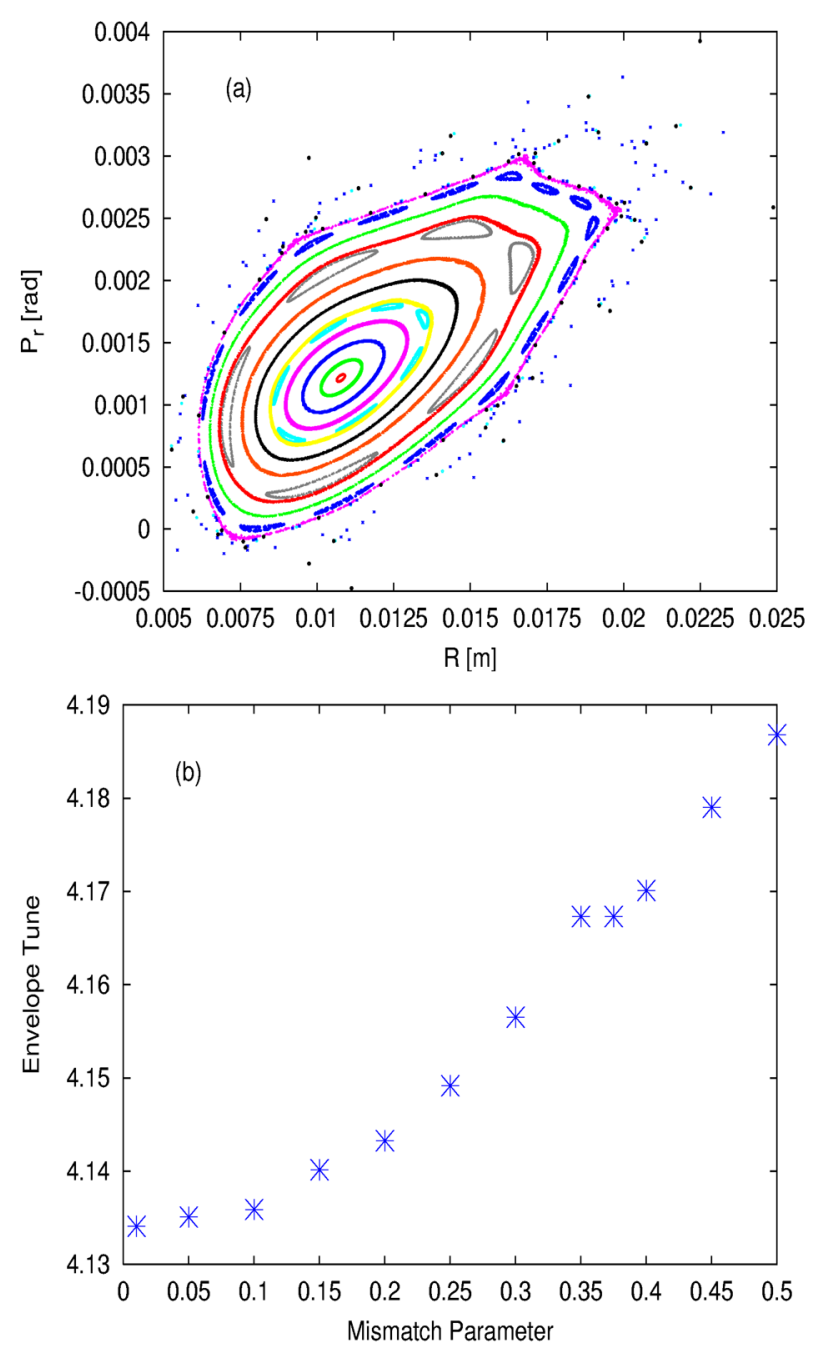

FIG. 9. (Color) (a) Stroboscopic map of the envelope in the PSR lattice for the full PSR beam intensity listed in Table I and emittances representative of the PSR beam at this intensity. The colors represent varying levels of mismatch. (b) Envelope tune versus mismatch.

instability in the real focusing structure. However, the altered island structure and lack of stochasticity result in different envelope dynamics. Thus for the mismatched beam the uniform-focusing approximation is incomplete.

The last issue of consideration for the mismatched beam envelope is the effect of dispersion on the resonance structure. A case with particularly strong resonance structure at $\Delta p / p=0$ is chosen, and the same parameters are used to recreate the map with $\Delta p / p=0.001$. Figure 11 shows the result, where the top plot is the zero momentum spread case [Fig. 11(a)] containing a strong 3:1 resonance, and the plot immediately below is for $\Delta p / p=0.001$ [Fig. 11(b)]. The dispersion term in the envelope equation with $\Delta p / p=0.001$ produces a smaller envelope tune shift by $\Delta \nu_{e} \cong 0.002$, and thus the envelope tune has not yet reached the third order resonance. An increase in the beam intensity by $7 \%$ for the $\Delta p / p=$ 0.001 case [Fig. 11(c)] results in a reappearance of the 3:1
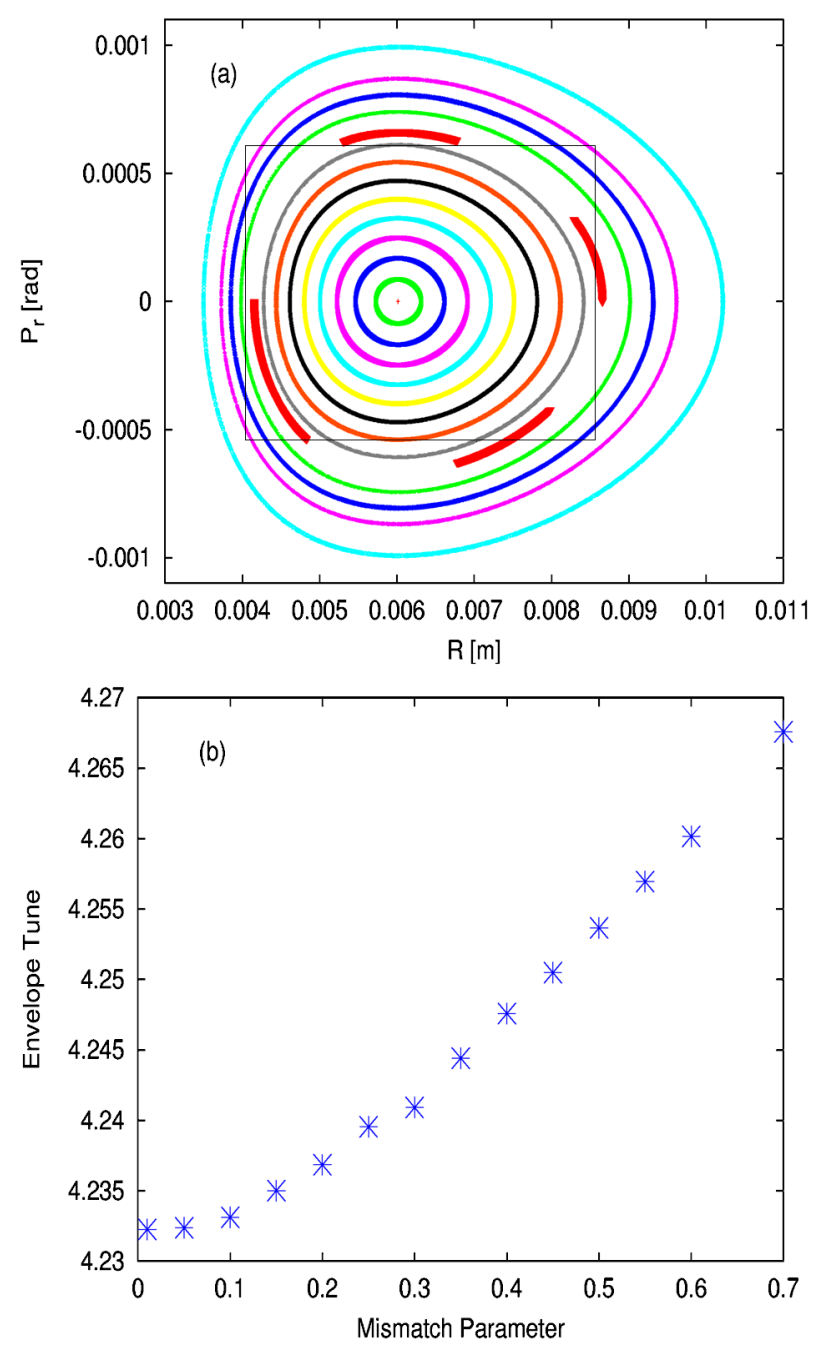

FIG. 10. (Color) (a) Stroboscopic map of the envelope in the PSR phase-advance-equivalent uniform-focusing lattice for a beam with intensity equal to one-quarter of the full PSR beam intensity listed in Table I. The colors represent varying levels of mismatch. (b) Envelope tune versus the mismatch values used in (a).

structure and envelope tunes that again lie on the resonance. Recall that for the closed-orbit resonance discussed in the previous section, the dispersion was beneficial to the beam because it decreased the space charge strength and the envelope resonance response. In contrast, there is no obvious benefit of dispersion to the mismatched envelope; the shift in envelope tune can bring the beam closer to a dangerous resonance as easily as it can move it away from one. However, the effect can be beneficial if the beam resides near the closed-orbit instability.

\section{THE ENVELOPE-PARTICLE PARAMETRIC RESONANCE}

In addition to the envelope dynamics of the beam, the space charge effect on single particles is also of interest, 

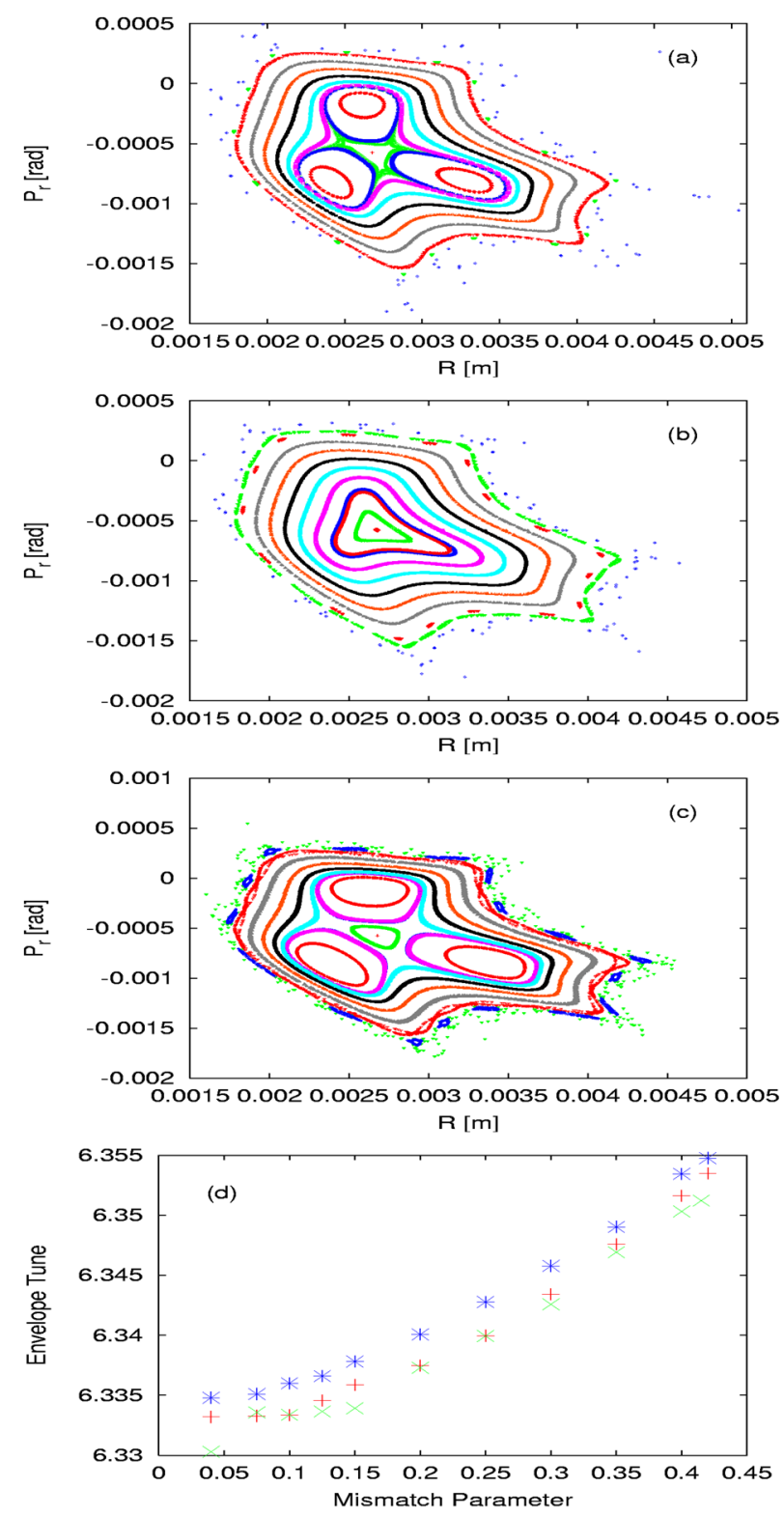

FIG. 11. (Color) (a) Horizontal stroboscopic map of a beam with $1.4 \times 10^{13}$ protons and $d p / p=0$. (b) Stroboscopic map of a beam with $1.4 \times 10^{13}$ protons and $d p / p=0.001$. (c) Stroboscopic map of a beam with $1.5 \times 10^{13}$ protons and $d p / p=0.001$. (d) Envelope tune versus mismatch parameter for the three cases above; the red +'s represent the beam with $1.4 \times 10^{13}$ protons and $d p / p=0$, the blue $*$ 's represent the beam with $1.4 \times 10^{13}$ protons and $d p / p=0.001$, and the green $\times$ 's represent the beam with $1.5 \times 10^{13}$ protons and $d p / p=$ 0.001 .

mainly because emittance growth depends on single particle processes. Though particle core model calculations which solve the envelope equations of motion cannot directly address emittance growth, it is possible to gain insight into some mechanisms of emittance growth by calculating the space charge force on single particles subjected to the core potential. Single particle behavior under the influence of the core can be sampled both inside and outside of the core boundary. The assumption here is that particles react to the field of the core, but the core does not react to the field of the particles. Studies of single particle dynamics in the KV framework indicate that halo can be produced via resonant interactions between the envelope core and particles that exist in a finite tail outside the core [4-7]. In our study, we track single particles under the influence of an elliptical KV potential using the equations:

$$
\begin{aligned}
& \Phi_{\text {inside }}=\frac{-4 Q e \lambda}{a b}\left[\cosh (2 u)+\cos (2 \theta)-e^{-[(1-b / a) /(1+b / a)]}\right. \\
& \times \cosh (2 u) \cos (2 \theta)]+K_{i}, \\
& \Phi_{\text {outside }}=-2 Q e \lambda u-Q e \lambda e^{-2 u} \cos (2 \theta)+K_{o}, \\
& x=\left(a^{2}-b^{2}\right)^{1 / 2} \cosh (u) \cos (\theta), \\
& y=\left(a^{2}-b^{2}\right)^{1 / 2} \sinh (u) \sin (\theta) .
\end{aligned}
$$

Here, $\Phi$ is the potential of the beam, $a$ and $b$ are the envelope radii described by Eqs. (6) and (7), $x$ and $y$ are horizontal and vertical particle coordinates, respectively, $\lambda$ is the beam particle line density, $Q$ is the charge number, and the $K$ 's are constants. Again, the model was developed for a longitudinally coasting beam.

Figure 12 shows stroboscopic maps of the particle phase space for the same intensity and beam parameters used in Fig. 7 (see Table I). The particle phase coordinates are normalized to the statistical (space charge adjusted) Twiss parameters, such that the edge of the beam core is given by $y^{2}+p_{y}^{2}=1$, and the time modulation of the phase-space structure is eliminated by plotting the particle at consecutive maxima of the beam envelope. Three envelope mismatches are considered: a perfectly matched envelope, an envelope with a $2 \%$ mismatch, and an envelope with a $30 \%$ mismatch. For the zero mismatched case, particles inside and outside of the beam core follow similar phase-space trajectories, and no resonance structure is observed. With a small, finite mismatch of $2 \%$, the structure of the 2:1 parametric resonance begins to appear outside of the envelope core [Fig. 12(b)]. Particles traversing the island trajectories have tunes that are nearly one-half of the envelope tune, and therefore they perform approximately one complete betatron oscillation for every two envelope oscillations. This condition also occurs inside a $\mathrm{KV}$ beam core with zero space charge, where all particles oscillate with tunes equal to half of the envelope tune. In a charged beam, however, the amounts of space charge tune depression realized for the envelope and for a core particle are not the same (see Ref. [6]), thus the condition for the resonance, $\nu_{e}=2 \nu_{y}$, can only be met in the zero-space charge limit, and the resonance condition can never be satisfied inside the core. Therefore the 2:1 islands are always found outside the core (see Appendix A). 

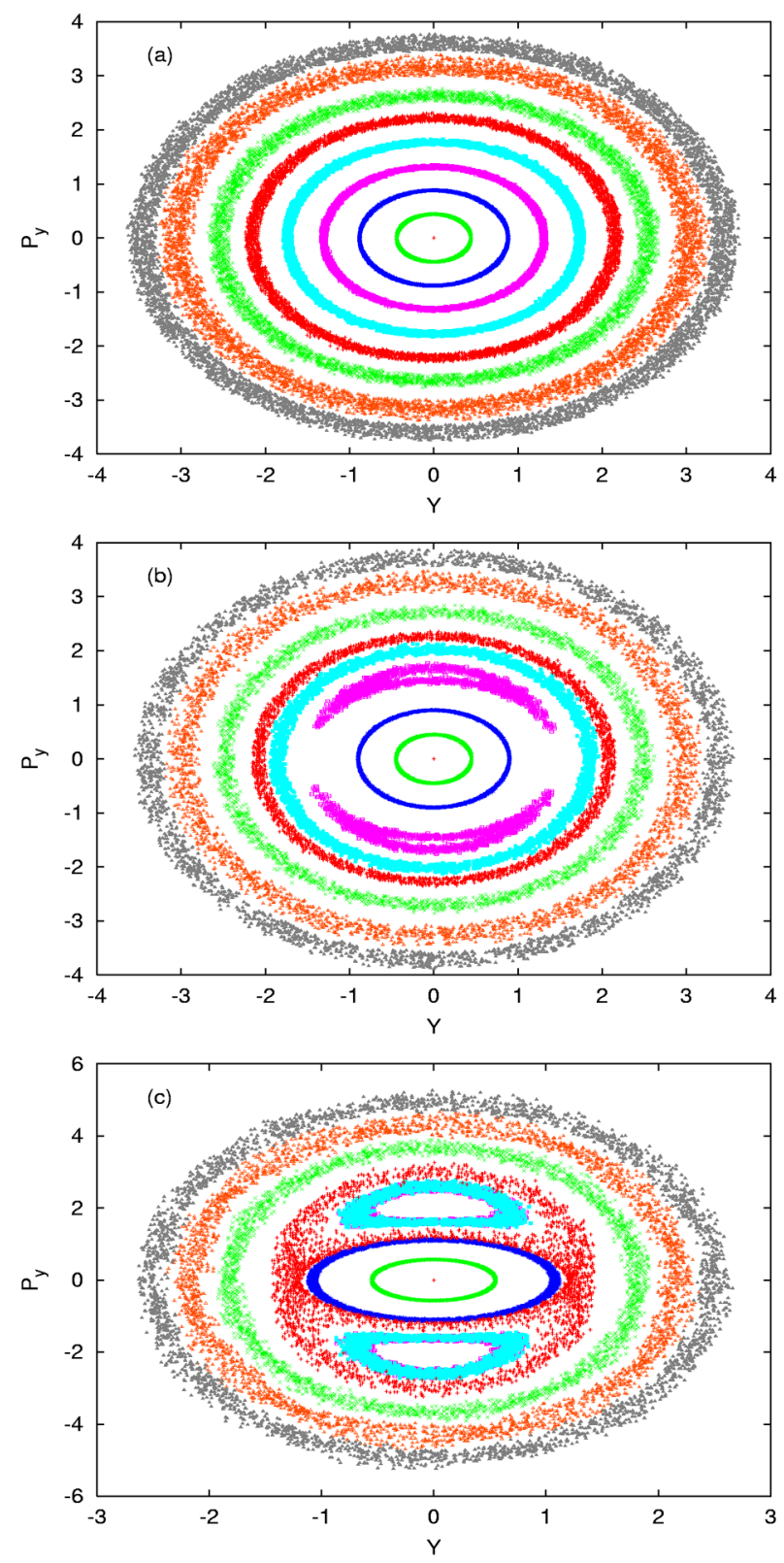

FIG. 12. (Color) (a) Stroboscopic map of the particle vertical phase space in the PSR lattice with zero envelope mismatch, $M=0$, and for a beam intensity equal to one-quarter of the full beam intensity listed in Table I. (b) Map for $M=0.02$. (c) Map for $M=0.30$. The particle coordinates are normalized to the statistical Twiss parameters, such that the core boundary is represented by $Y^{2}+P_{y}^{2}=1$.

In a KV beam, particles inside the core are bounded by the core potential, and thus particles that oscillate about the $2: 1$ resonance islands must be outside the core to begin with. These resonant particles have access to larger regions of phase space and are therefore defined as halo particles. The consequence of the resonance becomes more obvious at higher envelope mismatch, where the resonance islands become significantly broader and the stable fixed points move away from the core [Fig. 12(c)]. In Fig. 12, the stable fixed points for the $2 \%$ mismatch case reside at $P_{y} \cong 1.5$, and for the $30 \%$ mismatch case they move outward to $P_{y} \cong 2.0$. However, the migration of the stable fixed point away from the core does not decrease the accessibility of the resonance to particles just outside of the core boundary; rather, the growth of the island structure pushes the separatrix closer to the core boundary. Additionally, the unstable fixed points remain stationary over the range of mismatch. The overall effect of the increase in mismatch is to increase the potential for halo development.

An additional effect seen in the particle maps is the smearing of the phase-space trajectories. At higher intensities, the effect is even more pronounced. For example, stroboscopic maps for a beam at half of the full PSR intensity (double the intensity used in Fig. 12) and other parameters as listed in Table I, are shown for envelope mismatch of $2 \%$ and $30 \%$ in Fig. 13. The smearing of the trajectories can result from stochasticity, or from the dependence of the particle motion on both the frequency of the focusing structure and the frequency of the envelope motion. In the particle phase space, large degrees of stochasticity can lead to global chaos and emittance growth [6]. However, trajectory smearing due to the two-frequency effect is artificial and can be eliminated by an appropriate change of variables. Therefore, when considering the subject of emittance growth, it is important to know the contribution of the stochasticity to the smearing of the trajectories. The trajectory smearing is much smaller in the smooth approximation, as shown in Fig. 14, because the lattice effects are no longer present.

The level of stochasticity in a system can be assessed by measuring the rate of separation of two points with nearly identical initial conditions. When stochastic effects are strong, the separation between the two points will increase exponentially [20]. As an example, for two points located on the separatrix of Fig. 13(b) with separation less than 0.001 initially $\left(\sqrt{\left[\left(y_{2}^{2}-y_{1}^{2}\right)-\left(p_{y 2}^{2}-p_{y 1}^{2}\right)\right.} \cong 0.0007\right]$, the separation of the points versus longitudinal distance traveled by the beam is plotted in Fig. 15. It is clear that the two points do not move away from each other at an exponential rate before the distance begins to decrease again, as it must in a closed system. Examination of other regions of the phase space exhibited similar behavior, and thus stochasticity in the entire map is small. Unlike linac beams, where the space charge can be very large and global chaos can play an important role in emittance growth, the synchrotron beam has smaller space charge force and does not display this effect.

Though stochastic effects do not appear to be a strong candidate for emittance growth in the high intensity synchrotron beam, the $2: 1$ parametric resonance by itself can still cause emittance growth if the resonance islands are large and the separatrix is close to the beam core. As shown above, the conditions are met when the mismatch 

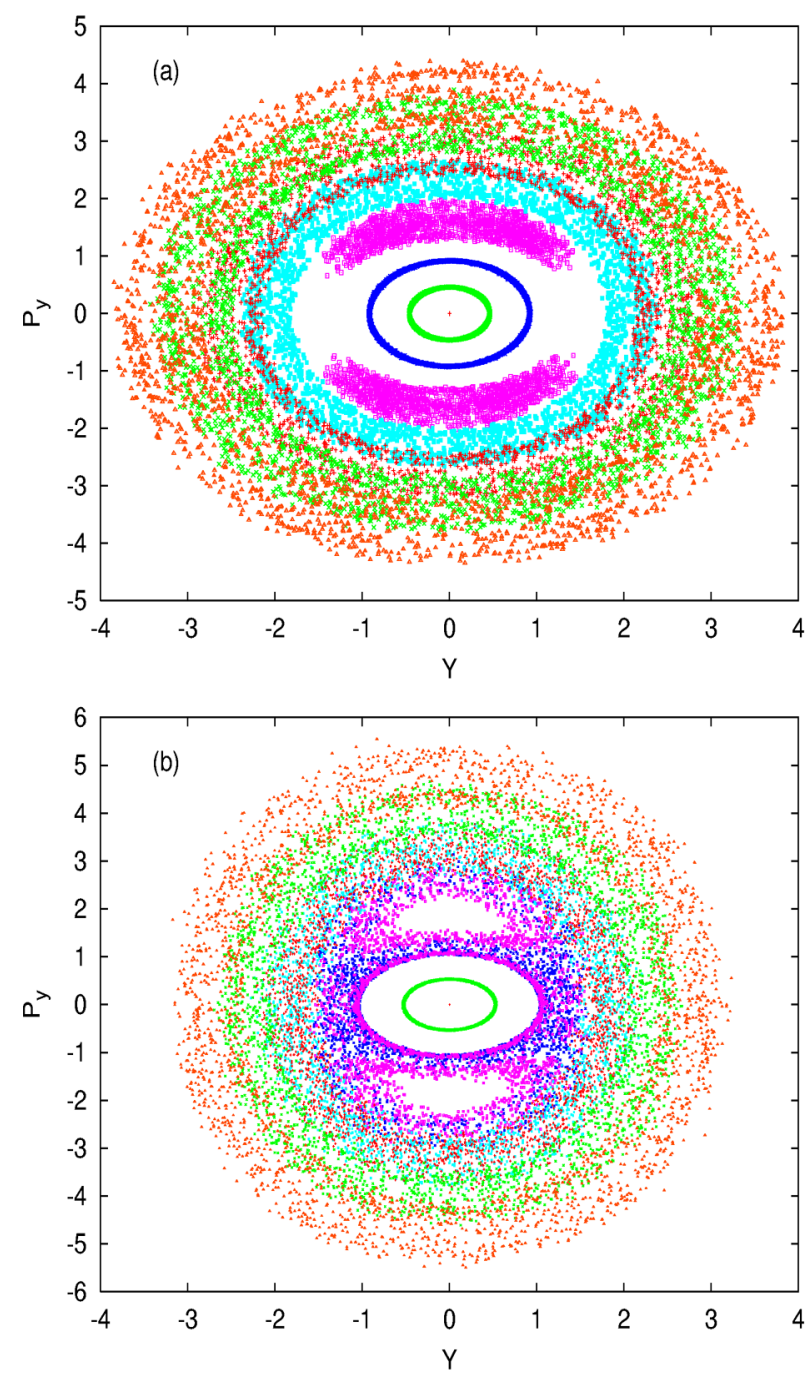

FIG. 13. (Color) (a) Stroboscopic map of the particle vertical phase space in the PSR lattice with envelope mismatch $M=$ 0.02 and for a beam intensity equal to one-half of the full beam intensity listed in Table I. (b) Map for $M=0.30$. The particle coordinates are normalized to the statistical Twiss parameters, such that the core boundary is represented by $Y^{2}+P_{y}^{2}=1$.

is large. However, a large mismatch is an unlikely condition in a synchrotron, and therefore the parametric resonance is not a probable source of emittance growth in the high intensity synchrotron beam. A more likely candidate for the emittance growth in the synchrotron beam is discussed in Appendix A.

\section{CONCLUSIONS}

A particle core model study has been conducted to explore the envelope resonance structure of a high intensity synchrotron beam. Specific to the PSR beam, our studies have shown strong likelihood of an integer $\nu_{e}=$ 4 envelope resonance in the vertical plane of the beam. For matched beam envelopes, it has been shown that the lattice harmonics drive resonant periodic envelope oscil-

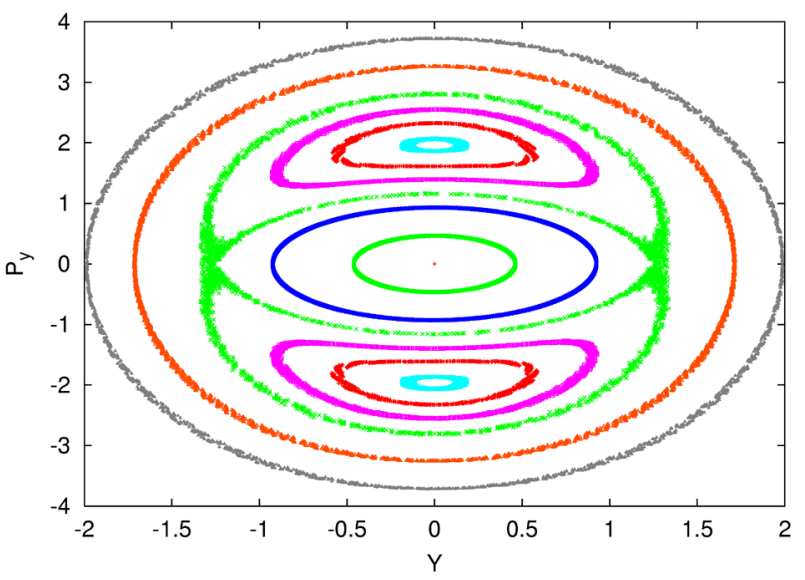

FIG. 14. (Color) Stroboscopic map of the particle vertical phase space in the uniform-focusing lattice with envelope mismatch $M=0.300$ and for a beam intensity equal to onehalf of the full beam intensity listed in Table I. The coordinates are normalized to the mismatched envelope.

lations. We have observed that the harmonic term necessary to drive the quadrupole-mode resonance in the vertical plane is contained within the bare lattice structure of the ring.

We have also investigated the effect of using a real lattice environment, including dispersion terms, on the envelope resonance behavior. For matched envelopes, we have observed that the lattice harmonics broaden the resonance response in both planes in a manner analogous to Sacherer's error driving terms, and that the dispersion provides the beneficial effect of decreasing the resonance response, especially for the particular plane that contains the dispersion. Furthermore, the dispersion masks the resonance signal in the envelope motion, making it difficult to study envelope resonances in a plane with dispersion.

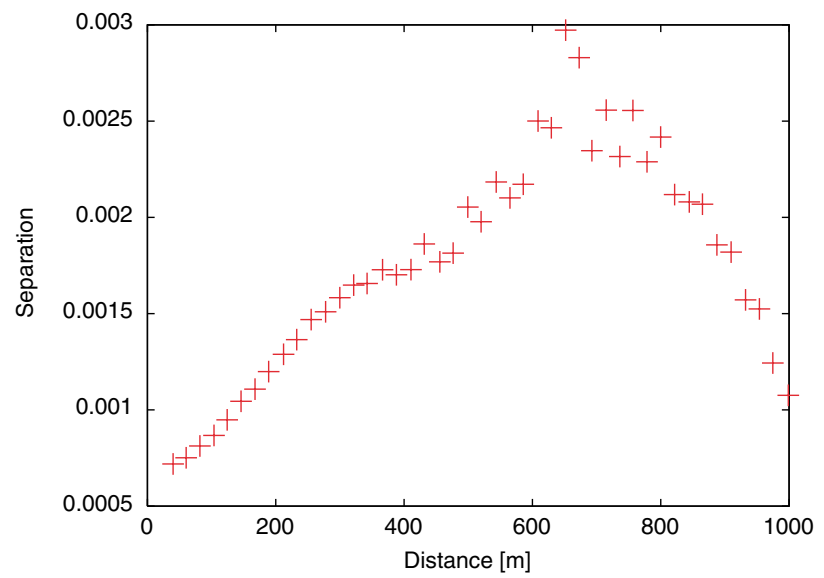

FIG. 15. (Color) Separation of two points located on the separatrix of Fig. 13 versus longitudinal distance traveled by the beam. 
The region surrounding the phase-space fixed point has been examined by tracking the motion of small oscillations about the envelope closed orbit. Complex phase-space behavior, including higher order resonance structure and large bands of stochasticity, has been observed. Finite $\Delta p / p$ in the mismatched beam increases the envelope tune slightly with no uniformly beneficial consequence. Moreover, we find that the uniform-focusing lattice is an insufficient approximation to the real lattice for the envelope evolution of a mismatched beam, giving nearly zero strength resonant islands and no stochasticity. For very high levels of space charge, where the tune of the mismatched envelope approaches integer values, the envelope encounters the closed-orbit instability if the lattice contains the appropriate driving harmonic.

Finally, the structure of the single particle phase space was studied. For the 2:1 envelope-particle parametric resonance, an increase in mismatch resulted in an increase in island size. The level of stochasticity in the maps was shown to be small, with the majority of the trajectory broadening resulting from the dependence of the particle motion on both the lattice focusing structure and the envelope motion. The 2:1 resonance structure can lead to beam halo when the islands are large and the separatrix is located close to the beam core. These conditions can be met for a highly mismatched beam. However, synchrotron beams are typically very close to being matched, and the resonance island is likely to be too small to produce emittance growth in this regime. Therefore, another mechanism must be responsible for the emittance growth observed in high intensity synchrotron beams.

\section{ACKNOWLEDGMENTS}

The authors would like to thank Robert Macek and the operations team at the LANSCE facility for providing us with experimental data and explanations of all parameters. We would also like to thank John Galambos for his initial motivation of this project. This work is supported by SNS through UT-Battelle, LLC, under Contract No. DE-AC05-00OR22725 for the U.S. DOE, and by Indiana University through grants from DOE, DEFG02-92ER40747 and NSF, PHY-0140251. The SNS is a partnership of six national laboratories: Argonne, Brookhaven, Jefferson, Lawrence Berkeley, Los Alamos, and Oak Ridge.

\section{APPENDIX A: THE PERIODIC ENVELOPE RESONANCE}

Consider a simple model of a 1D paraxial system, where the Hill's and envelope equations are given by

$$
\begin{cases}y^{\prime \prime}+\left[k(s)-\frac{K_{\mathrm{sc}}}{R_{\mathrm{b}}^{2}(s)}\right] y=0, & |y| \leq R_{\mathrm{b}}(s), \\ y^{\prime \prime}+k(s) y-\frac{K_{\mathrm{sc}}}{y}=0, & |y| \geq R_{\mathrm{b}}(s),\end{cases}
$$

$$
R_{\mathrm{b}}^{\prime \prime}+k(s) R_{\mathrm{b}}-\frac{\epsilon^{2}}{R_{\mathrm{b}}^{3}}-\frac{K_{\mathrm{sc}}}{R_{\mathrm{b}}}=0,
$$

where $y$ stands for either the horizontal or the vertical betatron coordinate of a particle, $k(s)$ is the focusing function, $K_{\mathrm{sc}}$ is the space charge perveance parameter, defined by $K_{\mathrm{sc}}=\left[\left(2 N r_{0}\right) /\left(\beta^{2} \gamma^{3}\right)\right], \epsilon=4 \epsilon_{\mathrm{rms}}$ is the $\mathrm{KV}$ beam emittance, $R_{\mathrm{b}}$ is the beam envelope radius, and $\beta(s)$ is the betatron amplitude function. A Floquet transformation can be performed with

$$
R=\frac{R_{\mathrm{b}}}{\sqrt{\beta(s) \epsilon}}, \quad \eta=\frac{y}{\sqrt{\beta(s)}}, \quad \phi=\frac{1}{\nu} \int_{0}^{s} \frac{d s}{\beta(s)} .
$$

Hill's and the envelope equations become

$$
\begin{gathered}
\ddot{\eta}+\nu^{2} \eta-\frac{\nu^{2} \beta(s) K_{\mathrm{sc}}}{\epsilon R^{2}} \eta=0, \\
\ddot{R}+\nu^{2} R-\frac{\nu^{2}}{R^{3}}-\frac{\nu^{2} \beta(s) K_{\mathrm{sc}}}{\epsilon R}=0,
\end{gathered}
$$

where $\nu$ is the betatron tune, and the overdots denote derivatives with respect to the new time coordinate, $\phi$.

For synchrotrons, the space charge terms in Hill's and the envelope equations can be considered as small perturbations unless a resonance is encountered. The envelope radius is expanded around the unperturbed closed orbit, i.e., the unperturbed betatron amplitude function, with $R=1+r+\Delta$, where $\Delta$ is a $\phi$-independent constant shift in the equilibrium radius and $r$ is the $\phi$-dependent term depending on the dynamics of the machine. Expanding the space charge term in a Fourier series:

$$
\frac{\nu \beta(s) K_{\mathrm{sc}}}{2 \epsilon}=\xi_{\mathrm{sc}}\left(1+\sum_{n=1}^{\infty} q_{n} \cos \left(n \phi+\chi_{n}\right)\right),
$$

where

$$
\xi_{\mathrm{sc}}=\frac{1}{4 \pi} \oint \frac{\beta(s) K_{\mathrm{sc}}}{\beta(s) \epsilon} d s
$$

is the Laslett (incoherent) linear space charge tune shift parameter, and $\xi_{\text {sc }} q_{n}$ is the Fourier amplitude of the $n$th harmonic, we find $\Delta \cong \xi_{\text {sc }} / 2 \nu$ and

$$
\ddot{r}+4\left(\nu^{2}-\nu \xi_{\mathrm{sc}}\right) r \approx 2 \nu \xi_{\mathrm{sc}} \sum_{n=1}^{\infty} q_{n} \cos \left(n \phi+\chi_{n}\right) .
$$

The space charge force plays two roles in the envelope equation: It decreases the envelope tune from $\nu_{\text {env }}=2 \nu$ to $\nu_{\mathrm{env}}=2\left(\nu-\frac{1}{2} \xi_{\mathrm{sc}}\right)$, and it generates a perturbation term, where the Fourier harmonic in the intrinsic betatron amplitude function serves as a harmonic perturbation to the envelope equation. The lattice harmonic expansion is more general than Sacherer's quadrupole error driving term in Eq. (1). The envelope radius, or the perturbed 


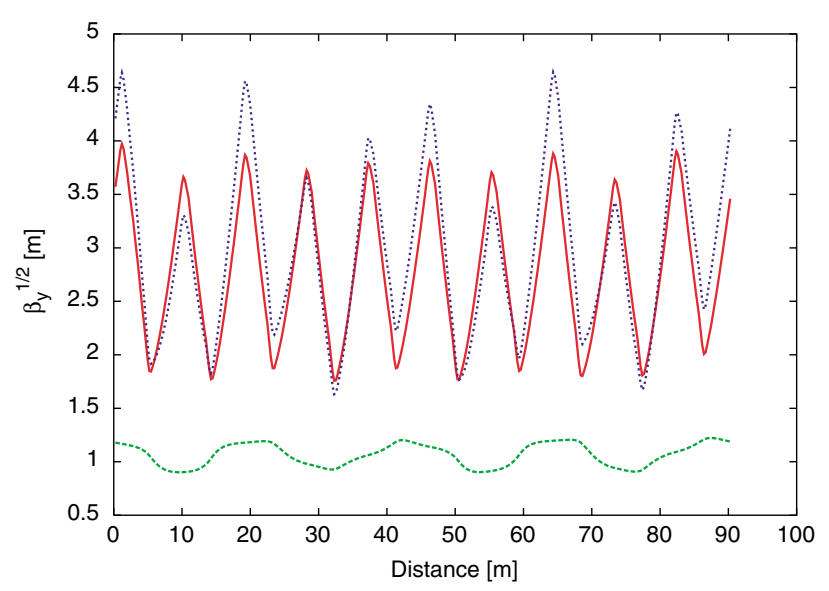

FIG. 16. (Color) The square root of the perturbed vertical betatron amplitude function (blue dashed line), for a beam with high intensity $\left(4.37 \times 10^{13}\right.$ particles $)$ in the PSR at LANL, is compared with the square root of the intrinsic betatron amplitude function (solid red line). The ratio of these two betatron amplitude functions, shown as the dashed (green) line, is the reduced envelope radius $R$ defined in Eq. (A3). Note that the average of $R$ is slightly larger than 1 .

betatron amplitude function, is resonantly excited by the harmonic $n \approx \nu_{\text {env }}$, with

$$
r \approx \frac{2 \nu \xi_{\mathrm{sc}} q_{n}}{-n^{2}+4\left(\nu^{2}-\nu \xi_{\mathrm{sc}}\right)} \cos \left(n \phi+\chi_{n}\right)
$$

Note that since the dependence of the space charge force on the envelope size has been ignored in Eq. (A9), the envelope radius becomes infinitely large when $n=2(\nu-$ $\left.\frac{1}{2} \xi_{\mathrm{sc}}\right)$. This is an artificial effect of the approximation, and a more accurate treatment of this problem is given in Sacherer's model [2].

Figure 16 shows the original betatron amplitude function (solid red line), the space charge perturbed vertical betatron amplitude function (green dashed line), and the normalized envelope radius $R$ (dotted blue line), obtained from a PIC simulation of the PSR at Los Alamos National Laboratory [11]. Since the vertical betatron tune of the PSR is about 2.19, the dominant perturbing harmonic in the envelope equation is 4 . There are clearly four oscillations in the normalized envelope radius $R$.

Inserting Eq. (A6) into Eq. (A4) and performing a similar analysis for the single particle motion inside the beam core, the following equation is obtained:

$$
\ddot{\eta}+\nu^{2} \eta-\frac{2 \nu \xi_{\mathrm{sc}}}{R^{2}}\left(1+\sum_{n=1}^{\infty} q_{n} \cos \left(n \phi+\chi_{n}\right)\right) \eta=0,
$$

where the particle tune is $\nu_{\mathrm{p}} \cong \nu-\xi_{\mathrm{sc}}$. Rightfully, $\xi_{\mathrm{sc}}$ is called the linear space charge tune shift parameter. One may speculate that a large envelope oscillation shown in Eq. (A9) may cause a large particle oscillation at $n=$ $2\left(\nu-\frac{1}{2} \xi_{\text {sc }}\right)$ resulting in a Mathieu instability $(2: 1$ para- metric resonance) [6]. However, by substituting $R=1+$ $\Delta+r$ into Eq. (A10), a more careful inspection shows that the resonance strength is actually zero, i.e., the envelope oscillation of the beam cannot affect particle motion inside the envelope. Particle motion inside the beam core cannot be resonantly excited. If particle motion inside the beam core is not affected by the envelope oscillation, what is the mechanism for emittance dilution?

Consider the situation that a beam, in equilibrium, circulates inside a synchrotron with space charge tune shift parameter $\xi_{\text {sc }}$. When new particles are injected into the beam core, the space charge parameter $\xi_{\text {sc }}$ increases, and the envelope tune is pushed toward an integer stop band $n \approx \nu_{e}$. If the stop band width $\xi_{\text {sc }} q_{n}$ is not zero, the envelope radius will be resonantly excited as shown in Eq. (A9). This causes a mismatch in the betatron phasespace ellipses for all particles inside and near the beam envelope radius. The emittance dilution may be caused by this phase-space mismatch, and further studies of this mechanism are underway.

[1] I. M. Kapchinskij and V.V. Vladimirskij, in Proceedings of the 9th International Conference on High Energy Accelerators, CERN, Geneva, Switzerland, 1959, edited by L. Kowarski (CERN, Geneva, 1959), p. 274.

[2] F. J. Sacherer, Ph.D. thesis, University of California, Berkeley, 1968.

[3] I. Hofmann, L. J. Laslett, L. Smith, and I. Haber, Part. Accel. 13, 145 (1983).

[4] R. L. Gluckstern, Phys. Rev. Lett. 73, 1247 (1994); A. Fedotov and R. L. Gluckstern, in Proceedings of the 1999 Particle Accelerator Conference, New York (IEEE, New York, 1999), p. 607, and references therein.

[5] T. P. Wangler et al., Phys. Rev. ST Accel. Beams 1, 084201 (1998).

[6] A. Riabko et al., Phys. Rev. E 51, 3529 (1995).

[7] J. A. Holmes, J. D. Galambos, D. Jeon, V.V. Danilov, and D. K. Olsen, Phys. Rev. ST Accel. Beams 2, 114202 (1999).

[8] George Lawrence, in Proceedings of the IEEE Particle Accelerator Conference, 1987, Washington, D.C. (IEEE, Piscataway, NJ, 1987), p. 825; D. Fitzgerald et al., in Proceedings of the 1999 Particle Accelerator Conference (Ref. [4]), p. 518.

[9] J. D. Galambos et al., Phys. Rev. ST Accel. Beams 3, 034201 (2000).

[10] J. A. Holmes et al., in Proceedings of the 2000 European Particle Accelerator Conference, Vienna (CERN, Geneva, 2000), p. 936.

[11] S. Cousineau et al., in Proceedings of the 2002 European Particle Accelerator Conference, Paris (EPS-IGA/ CERN, Geneva, 2002), p. 1016; S. Cousineau et al. (to be published).

[12] R. Baartman, in Workshop on Space Charge Physics in High Intensity Hadron Rings, AIP Conf. Proc. No. 448 (AIP, New York, 1998), p. 56. 
[13] A. Uesugi, S. Machida, and Yoshiharu Mori, Phys. Rev. ST Accel. Beams 5, 044201 (2002).

[14] A.V. Fedotov and I. Hofmann, Phys. Rev. ST Accel. Beams 5, 024202 (2002).

[15] S. Y. Lee and H. Okamoto, Phys. Rev. Lett. 80, 5133 (1998).

[16] J. Holmes, J. Galambos, D. Olsen, and S. Y. Lee, in Title (Ref. [12]), p. 254.
[17] F. Jones, Users' Guide to ACCSIM, TRIUMF Design Notes No. TRI-DN-90-17, 1990, http://www.triumf.ca/ compserv/accsim.html.

[18] T. Uesugi, S. Machida, and Y. Mori, Phys. Rev. ST Accel. Beams 5, 044201 (2002).

[19] S. Y. Lee et al., Phys. Rev. E 49, 5717 (1994).

[20] A. J. Lichtenberg and M. A. Lieberman, Regular and Stochastic Motion (Springer-Verlag, New York, 1983). 